Original Research Paper

\title{
Triton for Nuclear Fusion
}

\author{
${ }^{1}$ Raffaella Aversa, ${ }^{2}$ Relly Victoria Petrescu, ${ }^{1}$ Antonio Apicella, \\ ${ }^{3}$ Samuel Kozaitis, ${ }^{4}$ Taher Abu-Lebdeh, \\ ${ }^{5}$ Bilal Akash and ${ }^{2}$ Florian Ion Tiberiu Petrescu
}

${ }^{1}$ Advanced Materials Lab, Second University of Naples, Naples, Italy

${ }^{2}$ IFToMM, ARoTMM, Bucharest Polytechnic University, Bucharest, Romania

${ }^{3}$ Florida Institute of Technology, USA

${ }^{4}$ North Carolina A and T State University, USA

${ }^{5}$ Dean of School of Graduate Studies \& Research, American University of Ras Al Khaimah, UAE

Article history

Received: 10-11-2017

Revised: $07-12-2017$

Accepted: 16-12-2017

Corresponding Author: Florian Ion Tiberiu Petrescu IFToMM, ARoTMM, Bucharest Polytechnic University, Bucharest, Romania Email: scipub02@gmail.com
Abstract: In the nuclear fusion process that is permanently produced in the stars (suns) there is a thermonuclear reaction that uses as the main raw material the very first isotope of hydrogen, namely the Protium. This process is possible due to the huge temperatures and the unimaginably high pressures existing inside a star. At very high temperatures and pressures, matter begins to break even at the nuclear level. The nucleons split off and then reunited to form other types of nuclei. If it was initially thought that temperatures of tens or even hundreds of millions of degrees would be needed, today it is already proven that a minimum needed is about 40 trillion degrees. Such huge temperature is very difficult to be achieved on the Earth right now. For this reason, a compensatory solution would be the production of the nuclear fusion reaction with accelerated particles. For this reason, we want to express a major idea, namely the shift to the next hydrogen isotope, $3 \mathrm{H}$, Tritium, which is much less stable compared to the first two, with its widespread use for the achievement of nuclear-merging energy, here on the Earth. We can't achieve such temperatures yet, on earth, in safety, but especially to keep them. Only through dangerous bombs they can produce and maintain. Then the only method of achieving nuclear fusion power on the ground remains the use of particle accelerators. For this reason, modern physics power stations must look like or contain a nuclear particle accelerator. Whether we produce the cold or hot fusion reaction, we will need at least one particle accelerator. For a long time, I thought that Tokamak-type installations that have a toroidal shape represent the optimal solution for modern fusion power plants. Today, however, we doubt this, because the achieved tor has a small radius of action (the diameter of the tor is too small). But that is not the main issue that this paper proposes. In this study we want to propose the transition to experimentation of nuclear fusion energy, by exploiting (use) of tritium, namely the triton. The idea is to use triton nuclear fuel. But not the triton resulting from the presence of deuterium, but only pure triton, obtained from other methods than deuterium reactions. We propose, therefore, the elimination of deuterium as fuel and the use of Triton in its place.

Keywords: Nuclear Fusion, Tritium, Triton, Hydrogen Isotopes, Nuclear Energy

(C) 2017 Raffaella Aversa, Relly Victoria Petrescu, Antonio Apicella, Samuel Kozaitis, Taher Abu-Lebdeh, Bilal Akash and Florian Ion Tiberiu Petrescu. This open access article is distributed under a Creative Commons Attribution (CC-BY) 3.0 


\section{Introduction}

The field of energy development is the field of activities aimed at obtaining all possible sources of energy from natural resources. All of these activities include the production of conventional, alternative and renewable energy sources, as well as the recovery and re-use of energy that would otherwise be scattered. Energy conservation actions and the whole range of energy efficiency measures reduce demand for energy development and can benefit society by improving environmental issues. A simple, permanent way of efficiency and reduction of world energy consumption was represented by the software. By introducing the software, energy consumption has dropped drastically, so we can assert with certainty that in the future and continually, the implementation of new, superior software in all areas of industrial and civilian development will continue to produce a certain decrease continuing global energy consumption. The new technologies that have been implemented have also led to a drastic reduction in energy consumption and to achieving a superior global energy efficiency.

All companies use energy for transport, production, lighting, heating, air conditioning and communication for industrial, commercial and domestic purposes. Energy resources can be classified as primary resources if the resource can be used substantially in its original form or as a secondary resource where the energy source needs to be transformed into a more convenient form. All non-renewable resources are already exhausted at this time significantly due to human use over time, which has led to more and more new energy sources being introduced. While renewable resources are produced through ongoing processes that can support unlimited human exploitation, that is to say, they also have the character of sustainability, classical resources are largely consumed. The issue of classical energy resources is not only their disappearance but also the fact that they have polluted the blue planet very much over time. It is hoped that through the increasingly quantitative use of new energy resources mankind will succeed to deploy the planet, which we all inhabit and that we must take care to arrange it properly, preserve it and transmit it to generations future.

Hundreds of thousands of people work in the energy industry. The conventional industry includes the oil industry, the natural gas industry, the electricity industry and the nuclear industry. The new energy industries include the renewable energy industry, which includes the production, distribution and sale of alternative and sustainable fuels.

Actual reactors use uranium-238, which is more common than uranium-235 required by conventional reactors (Halliday and Robert, 1966; Harold Urey, from Wikipedia; Hydrogen, from Wikipedia; Jones, 2008;
Kramer, 2011; Krane, 1987; Lucas and Unterweger, 2000; Moses et al., 2009; Petrescu et al., 2017a; 2017b; 2017c; 2017d; 2016a; 2016b; Petrescu and Calautit, 2016; Petrescu, 2014; 2012a; 2012b; Petrescu and Petrescu, 2014; Shultis and Faw, 2002; Tritium, From Wikipedia; Zerriffi, 1996).

Today energy is no longer a problem for humanity. We have managed to overcome the critical threshold, with consumption ever rising and the planet's energy resources seem to be exhausting very quickly, as we always refer only to the classic hydrocarbon. We have quickly succeeded in finding major, sustainable, renewable, cheap, friendly, non-polluting energy resources, such as solar energy, solar power plants of various types, or wind, sea and ocean waves, or tides, or energy obtained from the salinity difference of water or those extracted from hot water springs and more. The important thing is that the planet has moved well over the past 15 years and these new energies have begun to be deployed and exploited more and more often in increasing quantities. He has understood by all the necessary forces the priority of this action and she has started very well. It should also be remembered that sustainable energy is also nuclear. It first saved us from a terrible major crisis that shrouded mankind in the 1970s and 1980s. Nuclear fission energy that has been steadily deployed and used since the 1960s until today has been a major source of energy for the planet and still accounts for a considerable amount of energy even today. It was I could say a kind of necessary bad.

We have managed to overcome a major energy, economic and social crisis due to the implementation of global nuclear energy obtained through fission.

Today we can breathe easily and we can make a calculation, just how much nuclear fission energy is needed. Besides the fact that supplies of uranium and they are still sufficient, we still have the reserve to increase wind and solar power plants more and more to the detriment of the use and implementation of nuclear fission power plants.

No nuclear accidents have frightened us so much because they represent a small percentage in comparison to the accidents caused by classic hydro power plants. The problem is today a professional and ethical one, which forces us to choose first, preferentially, the implementation of renewable, friendly solar or wind power, because today we can do this and it would be a pity to not do it. Why should we further increase our nuclear fission power plants when we can meet the energy needs of modern solar or wind power plants? We started to solve all the technical problems that had arisen many years ago about solar and wind energy. Even storing them is no longer a real problem. Is it not better to pursue a natural pattern, such as that of plants, animals and humans, who extract their energies in small 
quantities, from solar resources in general, but also using chemical processes. More quantities of small energy sources are able to meet the energy needs of living matter without the need for nuclear energy intervention, which besides the advantage of being produced locally in huge giants has the great disadvantage of representing a permanent danger being practically a clock bomb, an imminent danger to humanity. Nowhere in nature we have no examples of extracting the use of energy in concentrated quantities to represent an imminent danger. Is it not our duty to follow this example too?

It is much more convenient to create nuclear plants that give us an enormous amount of concentrated energy that we then distribute and spread, but this energyproducing style is also a more dangerous one, not at all used in the environment. Nature models teach us how we should do the right thing, to produce energy in small, friendly quantities and to use it locally, or even to distribute it, or to consume it and then to distribute it wherever it is needed.

At first it was difficult to implement such technologies, but today they no longer represent a real problem for humanity. Photovoltaic cells are now the key to success, being highly evolved. So wind energy, wind farms are today a friend of hope, which we can count on more and more. If we use only part of the planet's winds to produce energy, we will have enough energy resources for the planet all the time. Not to mention the Sun which is practical an inexhaustible energy source for us. Virtually all energy sources have their direct or indirect origins in solar energy.

After all, this process started well and continued now very well. Why then do we use nuclear energy for a while?

Because the classical energies from oil and pollutants are at the end, because at this moment we still do not have enough solar and wind power plants to satisfy absolutely all the momentary energy needs of the planet, because when we want to conquer the cosmic space, we have a great need and of concentrated energies, or at other difficult moments, such as the imminent need to defend the planet, let's say.

So it is the case not to leave the nuclear power plants completely, but to exploit them carefully, carefully. Furthermore, we still need research studies and nuclear fusion.

As we have shown in various papers, this form of energy, made in stars, needs a huge temperature to slow down and maintain itself alone. The thermonuclear reaction requires much higher temperatures than initially thought (Petrescu et al., 2017c; 2017d; 2016a; Petrescu and Calautit, 2016; Petrescu, 2014; 2012a; 2012b; Petrescu and Petrescu, 2014).

We can't achieve such temperatures yet, on earth, in safety, but especially to keep them. Only through dangerous bombs they can produce and maintain. Then the only method of achieving nuclear fusion power on the ground remains the use of particle accelerators. For this reason, modern physics power stations must look like or contain a nuclear particle accelerator.

Whether we produce the cold or hot fusion reaction, we will need at least one particle accelerator.

For a long time, I thought that Tokamak-type installations that have a toroidal shape represent the optimal solution for modern fusion power plants. Today, however, we doubt this, because the achieved tor has a small radius of action (the diameter of the tor is too small).

But that is not the main issue that this paper proposes.

In this study we want to propose the transition to experimentation of nuclear fusion energy, by exploiting (use) of tritium, namely the triton.

The idea is to use triton nuclear fuel.

But not the triton resulting from the presence of deuterium, but only pure triton, obtained from other methods than deuterium reactions. We propose, therefore, the elimination of deuterium as fuel and the use of Triton in its place.

\section{Materials and Methods}

Hydrogen $(\mathrm{H})$ is the smallest existing element and at the same time the first in the Mendeleev's Table. It has the standard atomic weight: (1.00784, 1.00811), conventionally 1.008) and is found naturally in the form of one of its three natural isotopes, noted with $1 \mathrm{H}, 2 \mathrm{H}$ and $3 \mathrm{H}$. The first two isotopes of hydrogen are stable (Fig. 1) (Halliday and Robert, 1966), i.e., Protium (1H) and Deuterium $(2 \mathrm{H})$, while the third isotope of hydrogen $(3 \mathrm{H})$ is unstable with a half-life of 12.32 years.

In the detail we added to the figure, one can easily see the three isotopes of the hydrogen in which the first two stable (Protium and Deuterium) are represented by black circles and the third unstable (Tritium) is represented by a white circle (on the first vertical line in detail).

All other heavier hydrogen isotopes that are known today are synthetic and have a half-life of fewer than 10 seconds (10-21 seconds). Of these, $5 \mathrm{H}$ is the most stable and $7 \mathrm{H}$ is the smallest (Lucas and Unterweger, 2000; Tritium, From Wikipedia).

The $2 \mathrm{H}$ (or hydrogen-2) isotope is commonly referred to as deuterium, while the $3 \mathrm{H}$ (or hydrogen-3) isotope is usually called tritium. Symbols $\mathrm{D}$ and $\mathrm{T}$ (instead of $2 \mathrm{H}$ and $3 \mathrm{H}$ ) are sometimes used for Deuterium and Tritium. The usual hydrogen isotope, the first, simplest and most common, neutron-free is sometimes (scientifically) called "Protium". Its ion (nucleus) is called Proton (isotope = Protium; ion = Proton). For Deuterium, symbol D or $2 \mathrm{H}$, the second isotope of hydrogen, if we remove it one electron one obtains its ion (nucleus) called Deuteron (isotope $=$ Deuterium; ion = Deuteron). For Tritium, symbol T or $3 \mathrm{H}$, the third isotope of hydrogen, if one remove one 
electron it obtains its ion (nucleus) called Triton (isotope $=$ Tritium; ion $=$ Triton). First two isotopes of hydrogen, Protium and Deuterium are stable and the third is unstable (see the first vertical line in the detail of Fig. 1). During the early radioactivity study, names were given to other heavy radioactive isotopes, but these names are rarely used today.

In the nuclear fusion process that is permanently produced in the stars (suns) there is a thermonuclear reaction that uses as the main raw material the very first isotope of hydrogen, namely the Protium.

This process is possible due to the huge temperatures and the unimaginably high pressures existing inside a star. At very high temperatures and pressures, matter begins to break even at the nuclear level.

The nucleons split off and then reunited to form other types of nuclei. The man has always dreamed of doing something like that on the Earth. Unfortunately, the procedures are not easy to achieve. If it was initially thought that temperatures of tens or even hundreds of millions of degrees would be needed, today it is already proven that a minimum needed is about 40 trillions degrees.

A such huge temperature is very very difficult to be achieved on the Earth right now (Petrescu et al., 2016a; Petrescu and Calautit, 2016; Petrescu, 2012a). At one $\mathrm{keV}$ is necessary a temperature of 10 million $^{0}$. At 400 $\mathrm{keV}$ (static calculations) is necessary a temperature of 4000 million $^{0}=4$ billions $^{0}$. At $4 \mathrm{GeV}=4000000 \mathrm{keV}$ (dynamic calculations) is necessary a temperature of 40000000 million $^{0}=40000$ billions $^{0}=40$ trillions $^{0}$ (Petrescu et al., 2016b; Petrescu et al., 2017a; 2017b; 2017c; 2017d).

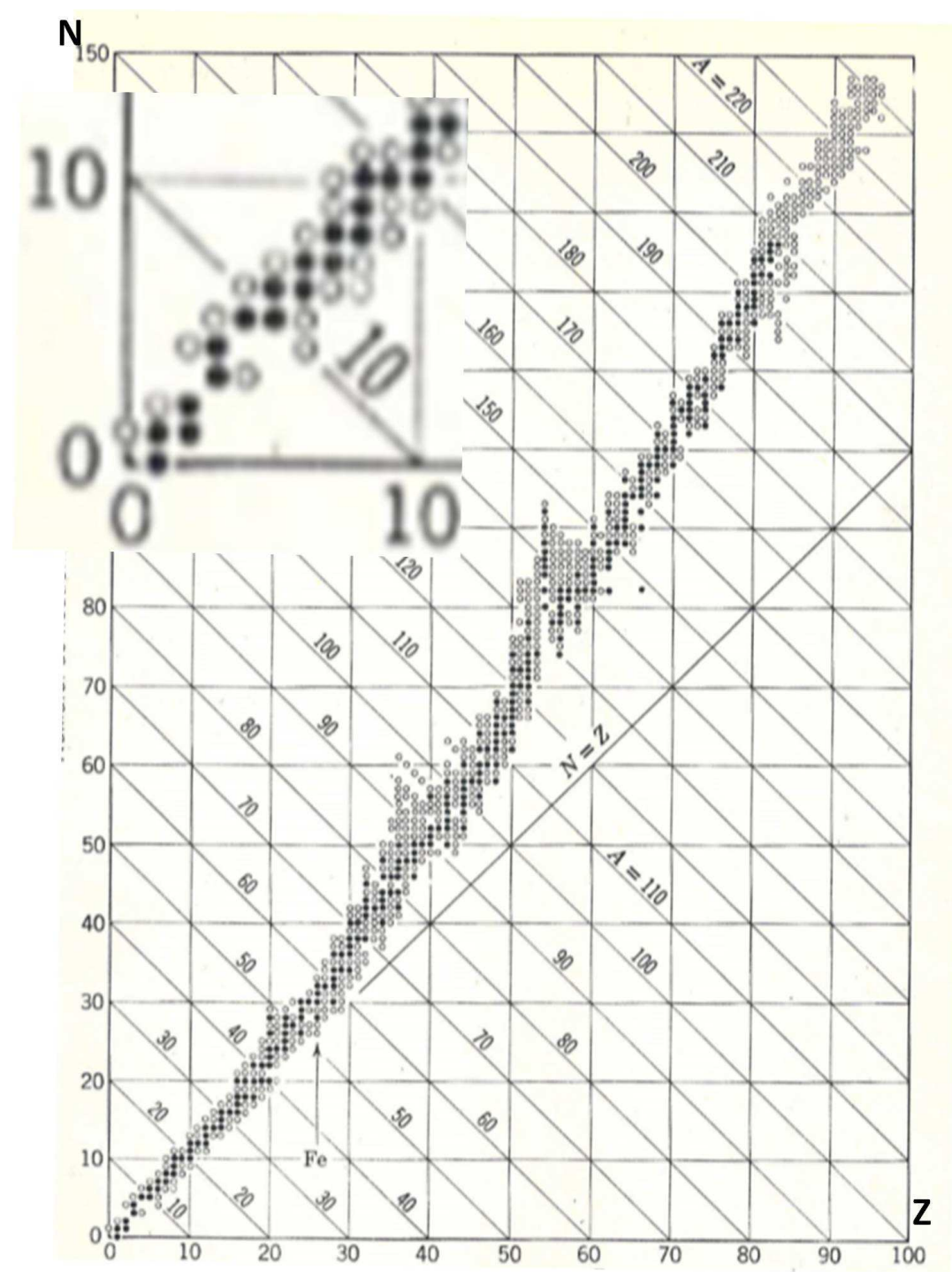

Fig. 1: Diagram of atomic cores (atomic nuclei). Source: (Halliday and Robert, 1966) 
Hardly temperatures of tens of millions and then hundreds of millions of degrees were obtained in the lab., but a such temperature of 40000000 of millions of degrees is very very difficult to be achieved today on the Earth.

For this reason, a compensatory solution would be the production of the nuclear fusion reaction with accelerated particles.

The second idea is to move on to a simpler reaction than the one that occurs in the stars.

The Protium is very stable. The Deuterium is also stable, but not as the Protium. The first thought of all the specialists was to move from Protium to Deuterium.

In this way, the work has been somewhat easier and it has always been thought that nuclear fusion has already taken place on earth with deuterium, but the dream has lasted for too long since the 1980s and has not yet materialized.

For this reason, we want to express a major idea, namely the shift to the next hydrogen isotope, $3 \mathrm{H}$, Tritium, which is much less stable compared to the first two, with its widespread use for the achievement of nuclear-merging energy, here on the Earth (Petrescu et al., 2016b; Petrescu et al., 2017a; 2017b; 2017c; 2017d).

Let talk about Tritium. Tritium (having the symbol T or $3 \mathrm{H}$ because it is also known as hydrogen-3) is a radioactive isotope of hydrogen. The tritium nucleus, ie tritium ion (called Triton) contains a proton and two neutrons, while the core of the antitumor (the most abundant hydrogen isotope) contains a proton and no neutron. Natural tritium is rarely found on Earth, where the amount of traces is formed by the interaction of the atmosphere with the cosmic rays. It can be produced by irradiating lithium ceramic pearls or metal lithium in a nuclear reactor. Tritium is sometimes used as a radioactive marker in radioluminescent light sources for instruments and watches and together with deuterium is used as a nuclear fuel for nuclear fusion reactions with applications in nuclear power generation as well as nuclear weapons. His name is derived from the Greek third (trítos), ie "the third" (Tritium, From Wikipedia).

While tritium has several different experimentally determined values of its half-life, the National Institute of Standards and Technology lists $4,500 \pm 8$ days (12.32 \pm 0.02 years). It decays into helium- 3 by beta decay as in this nuclear equation (Resulting in a helium isotope, an electron, an electronic antineutrino and an amount of energy of $18.6 \mathrm{keV})$ :

$$
{ }_{1}^{3} T \rightarrow{ }_{2}^{3} \mathrm{He}^{1+}+e^{-}+\bar{v}_{e}+18.6 k e V
$$

Tritium is produced in nuclear reactors by neutron activation of lithium- 6 . This is possible with neutrons of any energy and is an exothermic reaction yielding 4.8 $\mathrm{MeV}$. In comparison, the fusion of deuterium with tritium releases about $17.6 \mathrm{MeV}$ of energy. For applications in proposed fusion energy reactors, such as ITER, pebbles consisting of lithium bearing ceramics including $\mathrm{Li}_{2} \mathrm{TiO}_{3}$ and $\mathrm{Li}_{4} \mathrm{SiO}_{4}$, are being developed for tritium breeding within a Helium Cooled Pebble Bed (HCPB), also known as a breeder blanket:

$$
\begin{aligned}
& { }_{3}^{6} \mathrm{~L}+n \rightarrow{ }_{2}^{4} \mathrm{He}+2.05 \mathrm{MeV}+{ }_{1}^{3} \mathrm{~T}+2.75 \mathrm{MeV} \\
& ={ }_{2}^{4} \mathrm{He}+{ }_{1}^{3} \mathrm{~T}+4.784 \mathrm{MeV}
\end{aligned}
$$

High-energy neutrons can also produce tritium from lithium-7 in an endothermic (a net heat consuming reaction) reaction, consuming $2.466 \mathrm{MeV}$. This was discovered when the 1954 Castle Bravo nuclear test produced an unexpectedly high yield:

${ }_{3}^{7} \mathrm{~L}+n+2.466 \mathrm{MeV} \rightarrow{ }_{2}^{4} \mathrm{He}+{ }_{1}^{3} \mathrm{~T}+n$

Virtually a high energy neutron is introduced and is obtained another one of low energy (Zerriffi, 1996).

Using high energy neutrons to irradiate boron 10 , occasionally tritium and helium can be obtained (Jones, 2008):

${ }_{5}^{10} \mathrm{~B}+n \rightarrow 2{ }_{2}^{4} \mathrm{He}+{ }_{1}^{3} \mathrm{~T}$

\section{Results}

First-time Deuterium was extracted from water in 1931 by Harold Urey (Harold Urey, from Wikipedia). Small linear electrostatic accelerators have indicated that D-D reaction (fusion of two deuterium nuclei) is exothermic, even at that time.

It is known that not only the second isotope of hydrogen (Deuterium) can produce fusion nuclear energy, but and the third (heavy) isotope of hydrogen (Tritium) may produce energy through a nuclear fusion.

First nuclear fusion reaction it is possible between two nuclei of Deuterium and may be obtained: One Tritium nucleus plus a proton and energy, either a helium isotope with a neutron and energy (Equation 5 and 6), (Petrescu and Calautit, 2016; Petrescu, 2012a; Petrescu et al., 2016b; Petrescu et al., 2017a; 2017b; 2017c; 2017d; Petrescu, 2012b; Shultis and Faw, 2002; Petrescu and Petrescu, 2014; Petrescu, 2014):

$$
\begin{aligned}
& { }_{1}^{2} \mathrm{D}+{ }_{1}^{2} \mathrm{D} \rightarrow{ }_{1}^{3} \mathrm{~T}+1.01 \mathrm{MeV}+{ }_{1}^{1} \mathrm{H}+3.02 \mathrm{MeV} \\
& ={ }_{1}^{3} \mathrm{~T}+{ }_{1}^{1} \mathrm{H}+4.03 \mathrm{MeV} \\
& { }_{1}^{2} \mathrm{D}+{ }_{1}^{2} \mathrm{D} \rightarrow{ }_{2}^{3} \mathrm{He}+0.82 \mathrm{MeV}+{ }^{1} n+2.45 \mathrm{MeV} \\
& ={ }_{2}^{3} \mathrm{He}+{ }^{1} n+3.27 \mathrm{MeV}
\end{aligned}
$$

Fusion reaction may occur and between a nucleus of Deuterium and one of the Tritium (Equation 7) and this 
fusion nuclear reaction may be produced more easily than one between two deuterons (Equation 5 and 6):

$$
\begin{aligned}
& { }_{1}^{2} \mathrm{D}+{ }_{1}^{3} \mathrm{~T} \rightarrow{ }_{2}^{4} \mathrm{He}+3.5 \mathrm{MeV}+{ }^{1} n+14.1 \mathrm{MeV} \\
& ={ }_{2}^{4} \mathrm{He}+{ }^{1} n+17.6 \mathrm{MeV}
\end{aligned}
$$

An important nuclear reaction may be produced between a nucleus of Deuterium and an isotope of Helium (Equation 8):

$$
\begin{aligned}
& { }_{1}^{2} \mathrm{D}+{ }_{2}^{3} \mathrm{He} \rightarrow{ }_{2}^{4} \mathrm{He}+3.6 \mathrm{MeV}+{ }_{1}^{1} \mathrm{H}+14.7 \mathrm{MeV} \\
& ={ }_{2}^{4} \mathrm{He}+{ }_{1}^{1} \mathrm{H}+18.3 \mathrm{MeV}
\end{aligned}
$$

The isotope of helium is obtained in the reaction of 6 .

The reaction of the 5 generates Tritium which together with Deuterium (if one of them or both have enough energy) produce the nuclear reaction of 7 to generate a lot of energy and helium, a nontoxic, inert and very stable gas (Moses et al., 2009).

For this reason, the group of nuclear reactions of fusion is an advantageous one, friendly, pure, peacefully and inexpensive (Kramer, 2011; Krane, 1987).

Naturally, the Tritium appears in the nuclear reactor, only when the reaction of 1 is produced, but one may obtain more Tritium from the nuclear reaction (Equation 2):

The bars of lithium are easily entered or extracted in the nuclear reactor and by this mechanism can be controlled very simple and the fusion reaction speed at any time.

The reaction of the 9 can help much the reaction to the merger, by controlling its production. Lithium reserves in the earth's crust would permit the operation of melting plants for more than 1,000 years and those of the oceans could meet the needs of millions of years.

Neutrons necessary for producing the reaction 9 are generated even in the reactor in the framework of the reactions 6 and 7 .

Raw materials to achieve nuclear fusion are the deuterium and lithium, more exactly heavy water and bars of lithium. Result in a lot of energy and helium.

The reaction can be controlled easily through various methods. The reaction of the merger no tends to "get out of control" such as to the fission (being difficult to start it and easy to stop it) (Petrescu and Petrescu, 2014; Petrescu, 2014; Moses et al., 2009; Kramer, 2011; Krane, 1987).

Equation 9 can generate extra energy and it is much easier to achieve on the Earth than other possibilities:

$$
{ }_{1}^{3} \mathrm{~T}+{ }_{1}^{3} \mathrm{~T} \rightarrow{ }_{2}^{4} \mathrm{He}+2 \cdot{ }^{1} n+11.3 \mathrm{MeV}
$$

In a nuclear reactor of this type, supplied with Deuterium, can take place and other nuclear reactions, of which the most important are (Equation 10-12):
${ }_{2}^{3} \mathrm{He}+{ }_{2}^{3} \mathrm{He} \rightarrow{ }_{2}^{4} \mathrm{He}+2 \cdot{ }_{1}^{1} \mathrm{H}+12.9 \mathrm{MeV}$

${ }_{2}^{3} \mathrm{He}+{ }_{1}^{3} \mathrm{~T} \rightarrow{ }_{2}^{4} \mathrm{He}+{ }_{1}^{1} \mathrm{H}+{ }^{1} \mathrm{n}+12.1 \mathrm{MeV}$

${ }_{2}^{3} \mathrm{He}+{ }_{1}^{3} \mathrm{~T} \rightarrow{ }_{2}^{4} \mathrm{He}+4.8 \mathrm{MeV}+{ }_{1}^{2} \mathrm{D}+9.5 \mathrm{MeV}$

$={ }_{2}^{4} \mathrm{He}+{ }_{1}^{2} \mathrm{D}+14.3 \mathrm{MeV}$

Lithium with deuterium can still generate four other important reactions (Equation 13-16):

${ }_{1}^{2} \mathrm{D}+{ }_{3}^{6} \mathrm{Li} \rightarrow 2 \cdot{ }_{2}^{4} \mathrm{He}+22.4 \mathrm{MeV}$

${ }_{1}^{2} \mathrm{D}+{ }_{3}^{6} \mathrm{Li} \rightarrow{ }_{2}^{3} \mathrm{He}+{ }_{2}^{4} \mathrm{He}+{ }^{1} n+2.56 \mathrm{MeV}$

${ }_{1}^{2} \mathrm{D}+{ }_{3}^{6} \mathrm{Li} \rightarrow{ }_{3}^{7} \mathrm{Li}+{ }_{1}^{1} \mathrm{H}+5.0 \mathrm{MeV}$

${ }_{1}^{2} \mathrm{D}+{ }_{3}^{6} \mathrm{Li} \rightarrow{ }_{4}^{7} \mathrm{Be}+{ }^{1} \mathrm{n}+3.4 \mathrm{MeV}$

Lithium can react and with hydrogen $=$ Protium $(\mathrm{a}$ proton; Equation 17) or with an isotope of $\mathrm{He}$ (Equation 18):

${ }_{1}^{1} \mathrm{H}+{ }_{3}^{6} \mathrm{Li} \rightarrow{ }_{2}^{4} \mathrm{He}+1.7 \mathrm{MeV}+{ }_{2}^{3} \mathrm{He}+2.3 \mathrm{MeV}$

$={ }_{2}^{4} \mathrm{He}+{ }_{2}^{3} \mathrm{He}+4.0 \mathrm{MeV}$

${ }_{2}^{3} \mathrm{He}+{ }_{3}^{6} \mathrm{Li} \rightarrow 2 \cdot{ }_{2}^{4} \mathrm{He}+{ }_{1}^{1} \mathrm{H}+16.9 \mathrm{MeV}$

It should also be mentioned separately an extremely exciting nuclear reaction (Equation 19) that may occur between the stable isotope of boron with 6 neutrons (boron has five protons) and the first isotope of hydrogen, Protium (Hydrogen, from Wikipedia).

The reaction between hydrogen and boron can be achieved more easily than others and can generate a large amount of energy plus the inert gas, He:

${ }_{1}^{1} \mathrm{H}+{ }_{5}^{11} \mathrm{~B} \rightarrow 3 \cdot{ }_{2}^{4} \mathrm{He}+8.7 \mathrm{MeV}$

If the reaction of merger Protium-Protium can be produced only in the stars (as the Protium is a very stable isotope) on the Earth we can try the easiest to achieve the merger Tritium-Tritium (Equation 9), as the Tritium is an isotope unstable (see the diagram of the Fig. 1).

In the laboratory may be carried out and the reactions between Protium and Boron (Equation 19), or Protium and Lithium (Equation 17).

\section{Discussion}

The field of energy development is the field of activities aimed at obtaining all possible sources of energy from natural resources. All of these activities 
include the production of conventional, alternative and renewable energy sources, as well as the recovery and re-use of energy that would otherwise be scattered. Energy conservation actions and the whole range of energy efficiency measures reduce demand for energy development and can benefit society by improving environmental issues. A simple, permanent way of efficiency and reduction of world energy consumption was represented by the software. By introducing the software, energy consumption has dropped drastically, so we can assert with certainty that in the future and continually, the implementation of new, superior software in all areas of industrial and civilian development will continue to produce a certain decrease continuing global energy consumption. The new technologies that have been implemented have also led to a drastic reduction in energy consumption and to achieving a superior global energy efficiency.

All companies use energy for transport, production, lighting, heating, air conditioning and communication for industrial, commercial and domestic purposes. Energy resources can be classified as primary resources if the resource can be used substantially in its original form or as a secondary resource where the energy source needs to be transformed into a more convenient form. All non-renewable resources are already exhausted at this time significantly due to human use over time, which has led to more and more new energy sources being introduced. While renewable resources are produced through ongoing processes that can support unlimited human exploitation, that is to say, they also have the character of sustainability, classical resources are largely consumed.

The issue of classical energy resources is not only their disappearance but also the fact that they have polluted the blue planet very much over time. It is hoped that through the increasingly quantitative use of new energy resources mankind will succeed to deploy the planet, which we all inhabit and that we must take care to arrange it properly, preserve it and transmit it to generations future.

Hundreds of thousands of people work in the energy industry. The conventional industry includes the oil industry, the natural gas industry, the electricity industry and the nuclear industry. The new energy industries include the renewable energy industry, which includes the production, distribution and sale of alternative and sustainable fuels.

First-time Deuterium was extracted from water in 1931 by Harold Urey (Harold Urey, from Wikipedia). Small linear electrostatic accelerators have indicated that D-D reaction (fusion of two deuterium nuclei) is exothermic, even at that time.
It is known that not only the second isotope of hydrogen (Deuterium) can produce fusion nuclear energy, but and the third (heavy) isotope of hydrogen (Tritium) may produce energy through a nuclear fusion.

First nuclear fusion reaction it is possible between two nuclei of Deuterium and may be obtained: One Tritium nucleus plus a proton and energy, either a helium isotope with a neutron and energy (Equation 5 and 6).

The isotope of helium is obtained in the reaction of 6 .

The reaction of the 5 generates Tritium which together with Deuterium (if one of them or both have enough energy) produce the nuclear reaction of 7 to generate a lot of energy and helium, a nontoxic, inert and very stable gas (Moses et al., 2009).

For this reason, the group of nuclear reactions of fusion is an advantageous one, friendly, pure, peacefully and inexpensive (Kramer, 2011; Krane, 1987).

Naturally, the Tritium appears in the nuclear reactor, only when the reaction of 1 is produced, but one may obtain more Tritium from the nuclear reaction (Equation 2).

The bars of lithium are easily entered or extracted in the nuclear reactor and by this mechanism can be controlled very simple and the fusion reaction speed at any time.

The reaction of the 9 can help much the reaction to the merger, by controlling its production. Lithium reserves in the earth's crust would permit the operation of melting plants for more than 1,000 years and those of the oceans could meet the needs of millions of years.

Neutrons necessary for producing the reaction 9 are generated even in the reactor in the framework of the reactions 6 and 7 .

Raw materials to achieve nuclear fusion are the deuterium and lithium, more exactly heavy water and bars of lithium. Result in a lot of energy and helium.

The reaction can be controlled easily through various methods.

The reaction of the merger no tends to "get out of control" such as to the fission (being difficult to start it and easy to stop it).

After all, this process started well and continued now very well. Why then do we use nuclear energy for a while?

Because the classical energies from oil and pollutants are at the end, because at this moment we still do not have enough solar and wind power plants to satisfy absolutely all the momentary energy needs of the planet, because when we want to conquer the cosmic space, we have a great need and of concentrated energies, or at other difficult moments, such as the imminent need to defend the planet, let's say.

So it is the case not to leave the nuclear power plants completely, but to exploit them carefully, carefully. Furthermore, we still need research studies and nuclear fusion. 
As we have shown in various papers, this form of energy, made in stars, needs a huge temperature to slow down and maintain itself alone. The thermonuclear reaction requires much higher temperatures than initially thought (Petrescu et al., 2017c; 2017d; 2016a; Petrescu and Calautit, 2016; Petrescu, 2014; 2012a; 2012b; Petrescu and Petrescu, 2014).

We can't achieve such temperatures yet, on earth, in safety, but especially to keep them. Only through dangerous bombs they can produce and maintain. Then the only method of achieving nuclear fusion power on the ground remains the use of particle accelerators. For this reason, modern physics power stations must look like or contain a nuclear particle accelerator.

Whether we produce the cold or hot fusion reaction, we will need at least one particle accelerator.

\section{Conclusion}

In the nuclear fusion process that is permanently produced in the stars (suns) there is a thermonuclear reaction that uses as the main raw material the very first isotope of hydrogen, namely the Protium.

This process is possible due to the huge temperatures and the unimaginably high pressures existing inside a star. At very high temperatures and pressures, matter begins to break even at the nuclear level.

The nucleons split off and then reunited to form other types of nuclei.

If it was initially thought that temperatures of tens or even hundreds of millions of degrees would be needed, today it is already proven that a minimum needed is about 40 trillion degrees. Such huge temperature is very very difficult to be achieved on the Earth right now.

For this reason, a compensatory solution would be the production of the nuclear fusion reaction with accelerated particles. For this reason, we want to express a major idea, namely the shift to the next hydrogen isotope, $3 \mathrm{H}$, Tritium, which is much less stable compared to the first two, with its widespread use for the achievement of nuclear-merging energy, here on the Earth.

For a long time, I thought that Tokamak-type installations that have a toroidal shape represent the optimal solution for modern fusion power plants. Today, however, we doubt this, because the achieved tor has a small radius of action (the diameter of the tor is too small).

But that is not the main issue that this paper proposes.

In this study we want to propose the transition to experimentation of nuclear fusion energy, by exploiting (use) of tritium, namely the triton. The idea is to use triton nuclear fuel.

But not the triton resulting from the presence of deuterium, but only pure triton, obtained from other methods than deuterium reactions. We propose, therefore, the elimination of deuterium as fuel and the use of Triton in its place.

\section{Acknowledgement}

This text was acknowledged and appreciated by Dr. Veturia CHIROIU Honorific member of Technical Sciences Academy of Romania (ASTR) PhD supervisor in Mechanical Engineering.

\section{Funding Information}

Petrescu F.I., 2012 Cold nuclear fusion. Plasma Physics and Fusion Technology (S70), INIS 44(16).

\section{Author's Contributions}

All the authors contributed equally to prepare, develop and carry out this manuscript.

\section{Ethics}

Authors declare that are not ethical issues that may arise after the publication of this manuscript. This article is original and contains unpublished material.

\section{References}

Halliday, D. and R. Robert, 1966. Physics, Part II. 1st Edn., John Wiley and Sons, Inc., New York.

Harold Urey, from Wikipedia, the free encyclopedia. https://en.wikipedia.org/wiki/Harold_Urey

Hydrogen, from Wikipedia, the free encyclopedia. https://en.wikipedia.org/wiki/Hydrogen

Jones, G., 2008. Tritium issues in commercial pressurized water reactors. Fusion Sci. Technol., 54: 329-332. DOI: 10.13182/FST08-A1824

Kramer, D., 2011. DOE looks again at inertial fusion as a potential clean-energy source. Phys. Today, 64: 26-26. DOI: $10.1063 / 1.3563814$

Krane, K.S., 1987. Introductory Nuclear Physics. 3rd Edn., Wiley and Sons, New York, ISBN-10: 047180553X, pp: 864.

Lucas, L.L. and M.P. Unterweger, 2000. Comprehensive review and critical evaluation of the half-life of tritium. J. Res. Nat. Inst. Standards Technol., 105: 541-549. DOI: 10.6028/jres. 105.043

Moses, E.I., R.N. Boyd, B.A. Remington, C.J. Keane and R. Al-Ayat, 2009. The national ignition facility: Ushering in a new age for high energy density science. Phys. Plasmas, 16: 041006-041006. DOI: $10.1063 / 1.3116505$

Petrescu, F.I. and J.K. Calautit, 2016. About nano fusion and dynamic fusion. Am. J. Applied Sci., 13: 261-266. DOI: 10.3844/ajassp.2016.261.266

Petrescu, F.I., 2014. Nuclear fusion. Infinite Energy, 20: 44-47.

Petrescu, F.I., 2012a. Cold nuclear fusion. Plasma Phys. Fus. Technol., S70), INIS 44(16) 
Petrescu, F.I., 2012b Cold Nuclear Fusion. 1st Edn., Create Space Publisher, USA, ISBN-13: 978-1-4782-3426-5, pp: 80.

Petrescu, F.I., A. Apicella, R.V. Petrescu, S. Kozaitis and R. Bucinell et al., 2016a. Environmental protection through nuclear energy. Am. J. Applied Sci., 13: 941-946. DOI: 10.3844/ajassp.2016.941.946

Petrescu, R.V., R. Aversa, A. Apicella, S. Li and G. Chen et al., 2016b. Something about electron dimension. Am. J. Applied Sci., 13: 1272-1276. DOI: 10.3844/ajassp.2016.1272.1276

Petrescu, R.V., R. Aversa, S. Li, M.M. Mirsayar and R. Bucinell et al., 2017a. Electron dimensions. Am. J. Eng. Applied Sci., 10: 584-602. DOI: 10.3844/ajeassp.2017.584.602

Petrescu, R.V., R. Aversa, S. Kozaitis, A. Apicella and F.I.T. Petrescu, 2017b. Deuteron dimensions. Am. J. Eng. and Applied Sci., 10: 649-654. DOI: 10.3844/ajeassp.2017.649.654
Petrescu, R.V., R. Aversa, S. Kozaitis, A. Apicella and F.I.T. Petrescu, 2017c. Some proposed solutions to achieve nuclear fusion. Am. J. Eng. Applied Sci., 10: 703-708. DOI: 10.3844/ajeassp.2017.703.708

Petrescu, R.V., R. Aversa, S. Kozaitis, A. Apicella and F.I.T. Petrescu, 2017d. Some basic reactions in nuclear fusion. Am. J. Eng. Applied Sci., 10: 709-716. DOI: 10.3844/ajeassp.2017.709.716

Petrescu, F.I. and R.V. Petrescu, 2014. Nuclear green energy. IJAP, 10: 3-14.

Shultis, J.K. and R.E. Faw, 2002. Fundamentals of Nuclear Science and Engineering. 1st Edn., CRC Press, New York, ISBN-10: 0824708342, pp: 520.

Tritium, From Wikipedia, the free encyclopedia.

Zerriffi, H., 1996. Tritium: The environmental, health, budgetary and strategic effects of the department of energy's decision to produce tritium. Institute for Energy and Environmental Research. 